\title{
Femoroacetabular impingement syndrome and labral injuries: grading the evidence on diagnosis and non- operative treatment-a statement paper commissioned by the Danish Society of Sports Physical Therapy (DSSF)
}

\author{
Lasse Ishøi (D) ,' Mathias Fabricius Nielsen (D) ,' Kasper Krommes (D) ,' \\ Rasmus Skov Husted (D) , 2,3 Per Hölmich (D) , 1 Lisbeth Lund Pedersen (D) , \\ Kristian Thorborg (D) ${ }^{1}$
}

- Additional supplemental material is published online only. To view, please visit the journal online (http://dx.doi. org/10.1136/bjsports-2021104060).

For numbered affiliations see end of article.

\section{Correspondence to} Lasse Ishøi, Hvidovre Hospital, Sports Orthopaedic Research Center-Copenhagen (SORC-C), Arthroscopic Center, Department of Orthopedic Surgery, Copenhagen University Hospital, Amager-Hvidovre, Denmark, Kobenhavn, Denmark: lasse.ishoei@regionh.dk

Accepted 6 September 2021 Published Online First 16 September 2021

Check for updates

(C) Author(s) (or their employer(s)) 2021. No commercial re-use. See rights and permissions. Published by BMJ.

To cite: Ishøi $L$, Nielsen MF, Krommes K, et al. Br J Sports Med 2021:55:1301-1310.

\section{ABSTRACT}

This statement summarises and appraises the evidence on diagnostic tests and clinical information, and nonoperative treatment of femoroacetabular impingement (FAI) syndrome and labral injuries. We included studies based on the highest available level of evidence as judged by study design. We evaluated the certainty of evidence using the Grading of Recommendations Assessment Development and Evaluation framework. We found 29 studies reporting 23 clinical tests and 14 different forms of clinical information, respectively. Restricted internal hip rotation in $0^{\circ}$ hip flexion with or without pain was best to rule in FAl syndrome (low diagnostic effectiveness; low quality of evidence; interpretation of evidence: may increase post-test probability slightly), whereas no pain in Flexion Adduction Internal Rotation test or no restricted range of motion in Flexion Abduction External Rotation test compared with the unaffected side were best to rule out (very low to high diagnostic effectiveness; very low to moderate quality of evidence; interpretation of evidence: very uncertain, but may reduce post-test probability slightly). No forms of clinical information were found useful for diagnosis. For treatment of FAl syndrome, 14 randomised controlled trials were found. Prescribed physiotherapy, consisting of hip strengthening, hip joint manual therapy techniques, functional activity-specific retraining and education showed a small to medium effect size compared with a combination of passive modalities, stretching and advice (very low to low quality of evidence; interpretation of evidence: very uncertain, but may slightly improve outcomes). Prescribed physiotherapy was, however, inferior to hip arthroscopy (small effect size; moderate quality of evidence; interpretation of evidence: hip arthroscopy probably increases outcome slightly). For both domains, the overall quality of evidence ranged from very low to moderate indicating that future research on diagnosis and treatment may alter the conclusions from this review.

\section{INTRODUCTION}

Hip-related pain, typically affecting young and middle-aged individuals, ${ }^{1}{ }^{2}$ is associated with reduced physical activity $^{3}$ and poor quality of life. ${ }^{4}$ Based on imaging findings, hip-related pain is classified into (1) femoroacetabular impingement (FAI) syndrome, (2) acetabular dysplasia and/or hip instability and (3) cartilage and/or labral injury with normal bony morphology. ${ }^{5}$ FAI syndrome is the most common cause of hip-related pain, ${ }^{67}$ and is defined as a motion-related disorder of the hip joint caused by a collision between the head-neck junction of the femur with the acetabular rim due to cam and/or pincer morphology. ${ }^{1}$ This repetitive mechanical loading may result in acetabular labral ${ }^{8}$ and cartilage injuries. ${ }^{9-11}$

Consensus recommendations on diagnosis and treatment of patients with FAI syndrome/labral injuries have recently been published. ${ }^{15}{ }^{12}$ While these have been guided by findings from systematic reviews, ${ }^{13-15}$ rating of the overall quality of evidence using a contemporary framework, Grading of Recommendations Assessment, Development and Evaluation (GRADE), ${ }^{16}$ based on up-to-date risk of bias tools (risk of bias 2.0 for randomised controlled trial (RCT) studies and QUADAS-2 (A Revised Tool for the Quality Assessment of Diagnostic Accuracy Studies) for diagnostic studies) ${ }^{1718}$ is lacking. Since the GRADE level represents the confidence in the synthesised effect estimate, grading the evidence is the initial step towards developing clinical recommendations. ${ }^{16}$ To date, this has only been done for special tests concerning FAI syndrome, ${ }^{19}$ and for non-operative versus operative treatment ${ }^{20}$; however, the latter was not based on the risk of bias 2.0 tool. ${ }^{17}$ Furthermore, no summary of the utility of clinical information as a diagnostic tool is available. Consequently, this leaves general practitioners, sports physicians and physiotherapists-often the first healthcare professionals to see patients with hip and groin pain-with limited ability to judge the utility of diagnostic tests for labral injury, ${ }^{13}{ }^{15}$ clinical information, such as selfreported symptoms for diagnosis, ${ }^{121}$ and the effect of different non-operative treatment strategies. ${ }^{14}$ To aid clinicians in the management of patients with FAI syndrome/labral injuries, this commissioned statement by the Danish Society of Sports Physical Therapy (DSSF) provides a systematic evaluation concerning the diagnostic effectiveness of clinical 
tests and information, and the effect of non-operative treatment strategies.

\section{METHODS}

\section{Authors}

The authors were appointed by the DSSF and have different educational backgrounds (physiotherapists: LI, MN, KK, RH, KT, LLP; orthopaedic surgeon: PH; sports science: LI). LI, KT and $\mathrm{PH}$ have clinical and research expertise within the field of FAI syndrome through multiple scientific publications and daily treatment of patients non-operatively (LI and KT) and surgically $(\mathrm{PH})$. KK, KT, RH and LLP hold expertise within systematic search of literature, LI, MN and KK have expertise within grading of the evidence, while LI, KK, MN, RH and LLP have expertise with risk of bias assessments.

\section{Study design}

This statement concerns two domains: (1) diagnosis, including diagnostic tests and clinical information and (2) non-operative treatment of FAI syndrome/labral injuries. To deal with heterogeneity in inclusion criteria and evolving terminology across studies, we included studies that involved diagnoses of FAI, FAI syndrome, acetabular labral injuries or a combination. ${ }^{5}$ In addition, we included studies with patients defined as having hip joint-related pain ${ }^{22}$ if this was not purely due to osteoarthritis, dysplasia and so on. We excluded treatment studies concerning only surgical interventions and/or therapeutic hip injections, as this statement was commissioned by the DSSF, and neither of these treatments are practiced by sports physical therapists in Denmark. For simplicity and to facilitate use of contemporary terminology, ${ }^{1}$ studies using the terminology 'femoroacetabular impingement' will be referred to as FAI syndrome. We employed two separate systematic searches to identify literature for each domain, with inclusion of studies based on the highest level of available evidence. ${ }^{23}$ Data were synthesised and the quality of evidence was evaluated using the GRADE framework. ${ }^{16}$

\section{Literature search}

Two systematic searches covering (1) diagnostic tests and clinical information and (2) treatment were conducted in Medline (via PubMed), CENTRAL and Embase (via Ovid) in July 2020 and updated in July 2021. ${ }^{24}$ No restrictions were applied concerning the year of publication, however, only publications in English were included. We searched individual text words in title and abstract supplemented with $\mathrm{MeSH}$ or Entry terms if available. For both domains we included the population of interest (eg, "Femoroacetabular impingement [MeSH]") and combined this with test properties (eg, "sensitivity and specificity" [MeSH]) for the diagnosis domain, and with intervention (eg, "nonoperative" OR "conservative") and outcome (eg, "iHOT-33" OR "HAGOS") for the treatment domain. In addition, reference lists of the included studies and relevant systematic reviews were scanned for potential references. A flow chart of searches (online supplemental file 1) and the complete search strategy (online supplemental file 2) as supplementary.

\section{Selection of studies}

Identified studies from databases were extracted to Endnote (Clarivate Analytics, Philadelphia, Pennsylvania, USA) and automatically screened for duplicates. Subsequently, two authors (LI, MFN) performed a blinded screening of records to identify eligible studies. In line with a previous clinical statement paper, ${ }^{25}$ we included studies based on the highest level of available evidence. $^{23}$ This means that we initially screened for systematic reviews/meta-analyses of diagnostic studies and individual studies on diagnostic effectiveness for the diagnosis domain, and for systematic reviews/meta-analyses of RCTs and individual RCTs for the treatment domain, as these represent the highest starting point for the GRADE assessment. ${ }^{16}$ If no systematic reviews/ meta-analyses and/or RCTs were identified for treatment, we screened for observational studies. For the diagnosis domain, we aimed to include studies that compared clinical tests and/or clinical information, such as self-reported symptoms (ie, clicking, perceived restricted range of motion, etc) to either (1) diagnostic imaging, such as plain radiographs, MRI or arthrography (MRA) and CT, (2) intra-articular anaesthetic hip joint injection and/ or (3) surgery. For the treatment domain, we aimed to include studies that compared different forms of non-operative treatment approaches or compared non-operative treatment against surgery on self-reported hip function. In studies evaluating the treatment effect using several self-reported measures of hip function, we report outcomes from recommended patientreported outcome measures, ${ }^{1}{ }^{26}$ such as the Copenhagen Hip And Groin Outcome Score (HAGOS) ${ }^{27}$ and International Hip Outcome Tool-33 (iHOT-33), ${ }^{28}$ if available, or we report other patient-reported outcome measures, preferably related to sports function, if available (eg, Hip Osteoarthritis Outcome Score (HOOS)-Sport Subscale).

\section{Appraisal}

Two authors independently assessed risk of bias (LI and MFN) of individual studies, as required for the GRADE framework ${ }^{29}$ and in line with Cochrane procedures. In case of discrepancy between raters, a third assessor ( $\mathrm{RSH}$ ) was included to facilitate agreement. We used the Cochrane Collaboration's risk of bias assessment tool (version 2.0) for RCT's ${ }^{17}$ and QUADAS-2 tool for diagnostic studies. ${ }^{18}$ Furthermore, two authors independently assessed risk of bias (RSH and LLP) in systematic reviews using the ROBIS assessment tool. ${ }^{30}$ We chose risk of bias assessments rather than quality assessments in accordance with the Cochrane Collaboration, to reflect what extent the included studies should be believed as oppose to their methodological quality and reporting. ${ }^{31}$ If a systematic review/meta-analysis included a risk of bias assessment of individual studies using one of the assessment tools stated above, no further risk of bias assessment was conducted for these individual studies. However, if these tools were not used, we reassessed all risk of bias domains in the specific individual studies as part of this statement. This was the case for all studies included in the treatment domain.

\section{Data synthesis}

Two authors independently assessed the quality of evidence (LI and MFN) for each outcome related to diagnostic tests and clinical information (diagnostic effectiveness) and treatment (eg, hip function measured with iHOT-33) using the approach from the GRADE working group. ${ }^{16}$ Agreement was reached by consensus. The quality of evidence was graded as: (1) high certainty, indicating that further research is unlikely to change the confidence in the estimate of effect, (2) moderate certainty, indicating that further research is likely to have an important impact on confidence in the estimate of effect and may change the estimate, (3) low certainty, indicating that further research is very likely to have an important impact on the confidence in the estimate of effect and is likely to change the estimate or (4) certainty very low, indicating high uncertainty about the estimate. ${ }^{16}$ For treatment purposes, the starting quality of evidence was rated as 
'high' when data were based on RCTs. ${ }^{16}$ For diagnostic purposes, the starting quality of evidence was rated as high when based on cohort studies (prospective or cross-sectional). ${ }^{16}$ Subsequently, the quality of evidence could be downgraded one or two levels (eg, from high to moderate or low) for each of the following five domains of the GRADE approach: study limitations (ie, serious risk of bias such as lack of blinding of outcome assessor or other concerns determined to influence the study result), ${ }^{29}$ inconsistency (ie, the heterogeneity of the results across studies if more than one study was included for the specific outcome), ${ }^{32}$ indirectness (ie, poor generalisability of the findings to the target population, eg, uncertainty of the specific diagnosis due to inclusion criteria, use of a non-recommended patient-reported outcome measure, and/or uncertainty of the clinical value of a specific clinical test), ${ }^{33}$ imprecision of the estimates (ie, wide CIs) ${ }^{34}$ and risk of publication bias. ${ }^{35}$

To facilitate informative and unbiased communications of the findings, the interpretation of the findings was based on the recommendations from the GRADE Working Group, ${ }^{36}$ which includes a set of standardised statements based on the combined effect size and grading. ${ }^{36}$

\section{Diagnostic tests and clinical information}

We used positive $(\mathrm{LR}+)$ and negative $(\mathrm{LR}-$ ) likelihood ratios to assess the diagnostic effectiveness of clinical tests and clinical information in line with a previous statement paper $^{25}$ and best practice recommendations. ${ }^{37} 38$ LRs express the change in probability of the patient having the diagnosis and/or injury. ${ }^{37} 38$ An $\mathrm{LR}+>1$ increases the post-test probability of a diagnosis following a positive test, while an LR $-<1$ decreases the posttest probability of a diagnosis following a negative test. The diagnostic effectiveness of a positive and negative test was classified based on current guidelines as: very low (LR+: 1-2; LR-: $0.5-1$ ), low (LR+: $>2-5$; LR-: $0.2-<0.5$ ), moderate (LR+: $>5-10$; LR-: $0.1-<0.2)$; high $(\mathrm{LR}+:>10 ; \mathrm{LR}-$ : $<0.1) .{ }^{37}$ Diagnostic effectiveness of tests was downgraded due to imprecision of the estimates in cases where the 95\% CIs of the LRs encompassed at least two categories of diagnostic effectiveness (eg, 95\% CI ranging from very low to moderate diagnostic effectiveness in line with a previous statement paper. ${ }^{25}$

\section{Treatment effect}

We used standardised effect sizes (Hedges g) to determine the effect of treatment interventions in line with the Cochrane Collaboration. ${ }^{39}$ If this were not reported in included metaanalyses, we used Review Manager V.5.3 (The Nordic Cochrane Centre, Copenhagen) for the calculation to facilitate consistency of interpretation across studies. ${ }^{25}$ In such cases, we re-ran the analysis, if possible, using a random-effect model, unless otherwise stated in the original meta-analysis. ${ }^{40}$ Heterogeneity in study results was calculated using the $\mathrm{I}^{2}$ statistic, which is a measure to indicate the consistency of results across studies, from $0 \%$ (no inconsistency) to $100 \%$ (maximal inconsistency). ${ }^{41}$ For individual treatment studies not included in meta-analyses, we calculated Hedges g using a freely available Excel Sheet (Microsoft) applying between-group differences in change scores, if available, or else using between-group differences in follow-up scores. ${ }^{39}$ In both cases, Hedges g was calculated as an adjustment of Cohen's $\mathrm{d}^{42}$ using the correction factor $J=1-\frac{3}{4 \mathrm{df}-1} \cdot{ }^{43}$ The magnitude of treatment effect across studies and meta-analyses were assessed as trivial $(\mathrm{g}<0.2)$, small $(\mathrm{g} \geq 0.2)$, medium $(\mathrm{g} \geq 0.5)$ and large $(\mathrm{g} \geq 0.8) .^{42}$

\section{RESULTS}

In total, 57 $7^{613-15} 19202244-93$ studies were identified. For a detailed overview of risk of bias assessments, GRADE, and which individual studies are contained in systematic reviews, we refer to online supplemental file 3 .

\section{Domain 1: diagnostic tests and clinical information}

For diagnostic tests and clinical information, we identified 6 systematic reviews ${ }^{13} 1519$ 84-86 and 26 observational studies ${ }^{64-68}$ concerning diagnosis of FAI syndrome/labral injuries. One systematic review contained several meta-analyses of diagnostic effectiveness. ${ }^{13}$ The remaining systematic reviews did not provide additional information on diagnostic effectiveness above individual studies, and thus these were only used to retrieve risk of bias assessment if available. In total, we identified 23 clinical tests and 14 different forms of clinical information. Below we present only commonly used/studied diagnostic tests and tests with the best combined diagnostic effectiveness and quality of evidence (table 1). Diagnostic effectiveness of tests is presented within three categories: (1) FAI syndrome/labral injuries, (2) FAI syndrome and (3) labral injuries in accordance with reporting of the original literature. Clinical information was not found useful and is presented with a complete overview of diagnostic tests and their effectiveness in online supplemental file 4.

\section{Flexion Adduction Internal Rotation test}

One systematic review and meta-analysis ${ }^{13}$ and 16 cohort studies ${ }^{45-5256606163-656768}$ were included to investigate the diagnostic effectiveness of the Flexion Adduction Internal Rotation (FADIR) test. For diagnosis of FAI syndrome/labral injuries, two meta-analyses reported in one systematic review ${ }^{13}$ using MRA $^{5663-65}(\mathrm{n}=188)$ and surgery ${ }^{47606165}(\mathrm{n}=319)$ as reference standard, and one additional study ${ }^{50}(n=49)$ observed a moderate to very low diagnostic effectiveness (LR+: 0.86-1.04 and LR-: $0.14-2.3$; low to very low quality of evidence). For diagnosis of FAI syndrome, nine cohort studies ${ }^{45-49} 51526367$ $(n=693)$ observed a high to very low diagnostic effectiveness (LR+: $1.00-3.30$ and LR - : 0.09-0.83; low to very low quality of evidence). For diagnosis of isolated labral injuries, seven cohort studies ${ }^{49566061646568}(\mathrm{n}=325)$ observed a high to very low diagnostic effectiveness (LR+: 1.00-2.30 and LR-: 0.060.76 ; very low quality of evidence).

\section{Flexion Internal Rotation test}

One systematic review and meta-analysis ${ }^{13}$ and four cohort studies $^{6455566}$ were included to investigate the diagnostic effectiveness of the Flexion Internal Rotation (F-IR) test. For diagnosis of FAI syndrome, two cohort studies ${ }^{645}(\mathrm{n}=304)$ observed a very low diagnostic effectiveness (LR+: $1.25-1.51$ and $L R-$ : $0.68-0.73$; moderate quality of evidence). For diagnosis of labral injuries, one meta-analysis ${ }^{13}$ of two studies ${ }^{55}{ }^{66}(\mathrm{n}=27)$ and one additional study ${ }^{55}(\mathrm{n}=30)$ observed a moderate to very low diagnostic effectiveness (LR+: 1.10-1.28 and LR - : 0.15-0.23; very low quality of evidence).

\section{Flexion Abduction External Rotation test}

Seven cohort studies ${ }^{4455052-5456}$ were included to investigate the diagnostic effectiveness of the Flexion Abduction External Rotation (FABER) test. For diagnosis of FAI syndrome/labral injuries, three cohort studies ${ }^{44505}(\mathrm{n}=178)$ observed a very low diagnostic effectiveness (LR+: 0.73-1.10 and LR-: 0.72-2.20; low quality of evidence). For diagnosis of FAI syndrome, two cohort studies $^{45} 52(n=138)$ observed a very low diagnostic effectiveness 
Table 1 Diagnosis of femoroacetabular impingement syndrome/labral injury: effectiveness of clinical tests and grading the quality of evidence. 'Quality of evidence' refers to the overall quality of evidence for either positive or negative likelihood ratios across studies, whereas 'diagnostic effectiveness across studies' shows the range of diagnostic effectiveness (and number of patients) for studies of a specific test

\begin{tabular}{|c|c|c|c|c|c|c|}
\hline & \multirow[b]{2}{*}{ Likelihood ratio } & \multirow[b]{2}{*}{ Quality of evidence } & \multicolumn{4}{|c|}{ Diagnostic effectiveness across studies } \\
\hline & & & High & Moderate & Low & Very low \\
\hline \multicolumn{7}{|l|}{ FADIR test (pain provocation) } \\
\hline \multirow{2}{*}{$\begin{array}{l}\text { Based on two meta- } \\
\text { analyses }(n=188 \text { and } \\
n=319)^{13} \text { and one cohort } \\
\text { study }(n=49)^{50}\end{array}$} & $L R+=0.86-1.04$ & Low & & & & $n=556$ \\
\hline & $\mathrm{LR}-=0.14-2.3$ & Very low & & $n=319$ & $n=188$ & $\mathrm{n}=49$ \\
\hline \multicolumn{7}{|l|}{ FAI syndrome } \\
\hline \multirow{2}{*}{$\begin{array}{l}\text { Based on nine cohort } \\
\text { studies }(n=693)^{45-4951526367}\end{array}$} & $\mathrm{LR}+=1.00-3.30$ & Low & & & $n=69$ & $n=624$ \\
\hline & $\mathrm{LR}-=0.09-0.83$ & Very low & $n=35$ & $\mathrm{n}=94$ & $n=364$ & $\mathrm{n}=200$ \\
\hline \multicolumn{7}{|l|}{ Labral injury } \\
\hline \multirow{2}{*}{$\begin{array}{l}\text { Based on seven cohort } \\
\text { studies }(n=325)^{49566061} \\
646568\end{array}$} & $\mathrm{LR}+=1.00-2.30$ & Very low & & & $n=18$ & $\mathrm{n}=307$ \\
\hline & $\mathrm{LR}-=0.06-0.76$ & & $n=127$ & & $n=124$ & $\mathrm{n}=74$ \\
\hline \multirow{2}{*}{$\begin{array}{l}\text { Based on one meta-analysis } \\
(\mathrm{n}=27)^{13} \text { and one cohort } \\
\text { study }(\mathrm{n}=30)^{55}\end{array}$} & $\mathrm{LR}+=1.10-1.28$ & Very low & & & & $\mathrm{n}=57$ \\
\hline & $L R-=0.15-0.23$ & & & $n=27$ & $n=30$ & \\
\hline \multicolumn{7}{|l|}{ FABER test (pain provocation) } \\
\hline \multicolumn{7}{|l|}{ FAI syndrome } \\
\hline \multirow{2}{*}{$\begin{array}{l}\text { Based on two cohort studies } \\
(n=138)^{4552}\end{array}$} & $\mathrm{LR}+=0.79-0.87$ & Moderate & & & & $\mathrm{n}=138$ \\
\hline & $\mathrm{LR}-=1.21-1.14$ & & & & & $\mathrm{n}=138$ \\
\hline \multicolumn{7}{|c|}{ FABER test (restricted ROM) } \\
\hline \multicolumn{7}{|l|}{ FAl syndrome } \\
\hline \multirow{2}{*}{$\begin{array}{l}\text { Based on two cohort studies } \\
(\mathrm{n}=678)^{5254}\end{array}$} & $\mathrm{LR}+=1.01-1.36$ & Moderate & & & & $\mathrm{n}=678$ \\
\hline & $\mathrm{LR}-=0.41-0.93$ & & & & $\mathrm{n}=603$ & $\mathrm{n}=75$ \\
\hline \multicolumn{7}{|c|}{$\begin{array}{l}\text { Internal rotation in neutral hip position } \\
\text { (restrictedrange of motion) }\end{array}$} \\
\hline \multirow{2}{*}{$\begin{array}{l}\text { Based on one cohort studies } \\
(\mathrm{n}=63)^{45}\end{array}$} & $\mathrm{LR}+=4.83$ & Low & & & $n=63$ & \\
\hline & LR $-=0.76$ & Moderate & & & & $n=63$ \\
\hline
\end{tabular}

The diagnostic effectiveness of the positive (LR+) and negative (LR) likelihood ratios are classified individually as: very low (LR+: 1-2; LR-: 0.5-1), low (LR+:>2-5; LR-: 0.2$<0.5)$, moderate $(L R+:>5-10 ; L R-: 0.1-<0.2)$; high $(L R+:>10 ; L R-:<0.1)$.

FABER, Flexion Abduction External Rotation; FADIR, Flexion Adduction Internal Rotation; FAI, femoroacetabular impingement; F-IR, Flexion Internal Rotation.

when using pain provocation as a positive test (LR+: $0.79-0.87$ and LR - : 1.21-1.14; moderate quality of evidence), while two cohort studies $^{52}{ }^{54}(\mathrm{n}=678)$ observed a low to very low diagnostic effectiveness when using restricted range of motion as a positive test (LR+: 1.01-1.36 and LR-: 0.41-0.93; moderate quality of evidence) (table 1). For diagnosis of labral injury, one cohort study $^{56}(\mathrm{n}=18)$ observed a very low diagnostic effectiveness (LR+: 1.70 and LR-: 0.78; very low quality of evidence).

\section{Internal rotation in neutral hip position}

One cohort study ${ }^{45}(n=63)$ observed a very low to low diagnostic effectiveness for prone internal rotation in neutral $\left(0^{\circ}\right.$ hip flexion) hip position when using reduced range of motion as a positive test for diagnosing FAI syndrome (LR+: 4.83 and LR - : 0.76 ; low to moderate quality of evidence).

\section{Domain 2: treatment}

Eleven systematic reviews ${ }^{14} 20828387-93$ and $14 \mathrm{RCTs}^{22}$ 69-81 concerning treatment of FAI syndrome/labral injuries were identified. Two systematic reviews and meta-analyses compared different forms of non-operative treatment for FAI syndrome/ labral injuries, ${ }^{1490}$ and thus we included the most recent. ${ }^{14}$ In addition, seven systematic reviews and meta-analyses compared non-operative versus operative treatment ${ }^{20} 8283$ 87-89 92 ; however, since these were all based on the same three RCTs ${ }^{69-71}$ and thus provided almost identical results, we only included results from one meta-analysis. ${ }^{20}$ In addiction, one meta-analysis used inappropriate outcome measures and thus was not considered for inclusion. ${ }^{92}$ An overview of the content of the interventions is provided in table 2, while results are provided in table 3.

\section{Prescribed physiotherapy versus operative treatment}

A systematic review and meta-analysis, ${ }^{20}$ based on three RCTs $(\mathrm{n}=574),{ }^{69-71}$ showed a small effect and significant betweengroup difference on iHOT-33 at 8-12 months follow-up in favour of operative treatment for FAI syndrome (mean difference: 11.02 points, 95\% CI 4.83 to $17.21, \mathrm{I}^{2}=43 \%$, Hedges $\mathrm{g}=0.41)$ (moderate quality of evidence). Furthermore, one RCT $(n=80)$ also reported on 24 months follow-up, observing a small and non-significant between-group difference on iHOT-33 
Table 2 Short summary of interventions delivered in the included randomised controlled trial studies

\begin{tabular}{|c|c|c|}
\hline & Physiotherapy intervention & Comparator intervention \\
\hline \multicolumn{3}{|c|}{ Prescribed physiotherapy vs operative treatment } \\
\hline Griffin et al ${ }^{69}$ & $\begin{array}{l}\text { 6-10 physiotherapy sessions over } 12-24 \text { weeks. Content: education, assessment, } \\
\text { analgesics and exercise-based hip programme (muscle control/stability, } \\
\text { strengthening, stretching)+optional components. }\end{array}$ & $\begin{array}{l}\text { Arthroscopic hip surgery (routine practice) including } \\
\text { treatment of shape abnormalities, labral and cartilage } \\
\text { pathology and postoperative rehabilitation (usual care). }\end{array}$ \\
\hline Mansell et al ${ }^{70}$ & $\begin{array}{l}12 \text { physiotherapy sessions over } 6 \text { weeks. Content: joint mobilisations, soft tissue } \\
\text { mobility, stretching, therapeutic and motor control exercises. }\end{array}$ & $\begin{array}{l}\text { Arthroscopic hip surgery including acetabuloplasty, labral } \\
\text { repair/debridement and femoroplasty as indicated by } \\
\text { surgeon's judgement and } 6 \text { months postoperative physical } \\
\text { therapy. }\end{array}$ \\
\hline Palmer et al $\left.\right|^{129}$ & $\begin{array}{l}\text { Up to } 8 \text { physiotherapy sessions over } 5 \text { months. Content: advice, goal-based and } \\
\text { individually tailored programme focused on muscle strengthening, core stability } \\
\text { and movement control. }\end{array}$ & $\begin{array}{l}\text { Standardised arthroscopic hip surgery including } \\
\text { osteochodroplasty, labral repair or debridement, cartilage } \\
\text { lesion debridement and optional microfracture and } \\
\text { postoperative physiotherapy (routine care). }\end{array}$ \\
\hline Martin et $\left.a\right|^{81}$ & $\begin{array}{l}\text { At least } 24 \text { physiotherapy sessions over } 24 \text { weeks. Content: gait training, } \\
\text { range of motion training, strength training, patient education, manual therapy, } \\
\text { hydrotherapy, home exercise programme, functional strengthening, proprioception } \\
\text { and balance training. }\end{array}$ & $\begin{array}{l}\text { Arthroscopic hip surgery including acetabular labral } \\
\text { repair, femoroacetabular osteoplasty and } 24 \text { weeks of } \\
\text { postoperative physiotherapy. }\end{array}$ \\
\hline \multicolumn{3}{|c|}{ Prescribed physiotherapy vs passive modalities, stretching and/or advice } \\
\hline Smeatham et al ${ }^{17}$ & $\begin{array}{l}\text { Routine care +up to } 10 \text { physiotherapy sessions. Content: manual therapy, exercise- } \\
\text { based rehabilitation and gym-based group sessions. }\end{array}$ & Routine care. \\
\hline Kemp et $a l^{75}$ & $\begin{array}{l}\text { Eight physiotherapy ( } 30 \mathrm{~min}) \text { sessions+one supervised gym session }(30 \mathrm{~min})+2 \\
\text { weekly unsupervised exercise sessions over } 12 \text { weeks. Content: hip joint manual } \\
\text { therapy, hip and trunk muscle strengthening, functional activity-specific retraining } \\
\text { and education. }\end{array}$ & $\begin{array}{l}\text { Eight physiotherapy ( } 30 \mathrm{~min}) \text { sessions+one supervised gym } \\
\text { session }(30 \mathrm{~min})+2 \text { weekly unsupervised exercise session } \\
\text { over } 12 \text { weeks. Content: hip joint manual therapy, muscle } \\
\text { stretching and health education. }\end{array}$ \\
\hline Harris-Hayes et al ${ }^{73}$ & $\begin{array}{l}\text { Six physiotherapy }(60 \mathrm{~min}) \text { sessions and daily home exercises over } 6 \text { weeks. } \\
\text { Content: Task-specific training of basic functional tasks and patient-specific } \\
\text { symptom provoking tasks, hip muscle strengthening. }\end{array}$ & 6 week waiting period on wait list. \\
\hline \multicolumn{3}{|c|}{ Comparison between different physiotherapy interventions } \\
\hline Aoyama et $a l^{74}$ & $\begin{array}{l}\text { One education session }+8 \text { weeks daily home training }(20 \mathrm{~min}) \text { including hip muscle } \\
\text { training and trunk stabilisation training. }\end{array}$ & $\begin{array}{l}\text { One education session }+8 \text { weeks daily home training } \\
\text { ( } 20 \mathrm{~min} \text { ) including hip muscle training. }\end{array}$ \\
\hline Harris-Hayes et al ${ }^{22}$ & $\begin{array}{l}\text { Ten physiotherapy }(60 \mathrm{~min}) \text { sessions and daily home exercises over } 12 \text { weeks. } \\
\text { Content: task-specific training of basic functional tasks and patient-specific } \\
\text { symptom provoking tasks, hip muscle strengthening. }\end{array}$ & $\begin{array}{l}\text { Ten physiotherapy }(60 \mathrm{~min}) \text { sessions and daily home } \\
\text { exercises over } 12 \text { weeks. Content: lower extremity and } \\
\text { trunk strengthening, lower extremity flexibility. }\end{array}$ \\
\hline Wright et al2 & $\begin{array}{l}\text { Twelve physiotherapy }(60 \mathrm{~min}) \text { sessions+home exercise programme over } 6 \text { weeks. } \\
\text { Content: manual therapy, strengthening, stretching and neuromuscular/motor } \\
\text { control exercises. }\end{array}$ & $\begin{array}{l}\text { Advice, activity modification and a booklet with } 6 \text { exercises } \\
\text { addressing hip strength and flexibility. }\end{array}$ \\
\hline \multicolumn{3}{|c|}{ Prescribed post-operative physiotherapy vs advice } \\
\hline Kemp et $a l^{76}$ & $\begin{array}{l}\text { Eight physiotherapy ( } 30 \mathrm{~min} \text { ) sessions+home exercises } 4 \text { xweek over } 12 \text { weeks. } \\
\text { Content: manual hip joint and soft tissue mobilisation, hip muscle retraining, trunk } \\
\text { muscle retraining, functional, proprioceptive and sport-specific or activity-specific } \\
\text { retraining, enhancing physical activity, education and exercise manual. }\end{array}$ & $\begin{array}{l}\text { Eight physiotherapy ( } 30 \mathrm{~min} \text { ) sessions over } 12 \text { weeks. } \\
\text { Content: education, advice, education information sheets. }\end{array}$ \\
\hline Bennell et $a l^{78}$ & $\begin{array}{l}\text { Standard postoperative care }+7 \text { physiotherapy }(30 \mathrm{~min}) \text { sessions. Content: } \\
\text { education, manual therapy, deep hip rotator muscle strengthening, stretches, gym } \\
\text { and aquatic programme and return to sport guidance. }\end{array}$ & $\begin{array}{l}\text { Standard postoperative care +in-patient physiotherapy for } \\
\text { gait aid +written education material +requested to not } \\
\text { undertake formal rehabilitation programme. }\end{array}$ \\
\hline \multicolumn{3}{|c|}{ Preoperative physiotherapy vs massage therapy } \\
\hline Grant et $a l^{79}$ & $\begin{array}{l}\text { Two preoperative physiotherapy }(60 \mathrm{~min}) \text { sessions over } 8 \text { weeks }+3 \text { postoperative } \\
\text { sessions over } 12 \text { weeks. First session content: instruction on daily home exercises } \\
\text { and stretches, exercise booklet diary. Second to fifth session content: education, } \\
\text { advice, postoperative exercises massage, hydrotherapy and gait re-training. }\end{array}$ & $\begin{array}{l}\text { Two preoperative physiotherapy }(60 \mathrm{~min}) \text { sessions over } \\
8 \text { weeks }+3 \text { postoperative sessions over } 12 \text { weeks. First } \\
\text { session included massage therapy. The remaining } 4 \\
\text { sessions matched the intervention group. }\end{array}$ \\
\hline
\end{tabular}

Physiotherapy sessions are one-to-one/face-to-face otherwise it is noted.

in favour of operative treatment (mean difference: 6.3 points, $95 \%$ CI -6.1 to 18.7 , Hedges $\mathrm{g}=0.23$ ) (very low quality of evidence). ${ }^{70}$ For labral injury, one RCT $(n=90)$ in adults above 40 years old showed a medium effect and significant betweengroup difference on iHOT-33 at 12 months follow-up in favour of operative treatment (mean difference: 12.11 points, 95\% CI 3.27 to 20.96 , Hedges $g=0.61$ ) (moderate quality of evidence).

\section{Prescribed physiotherapy versus passive modalities, stretching and/} or advice

A systematic review and meta-analysis, ${ }^{14}$ based on two RCTs $(\mathrm{n}=54),{ }^{75} 77$ showed a medium effect and significant betweengroup difference on patient-reported function and pain (measured with iHOT-33 $3^{75}$ and Non-Arthritic Hip Score; NAHS $^{77}$ ) at 12 weeks follow-up in favour of prescribed physiotherapy for FAI syndrome (Hedges $\mathrm{g}=0.66$, 95\% CI 0.09 to $1.23, \mathrm{I}^{2}=0 \%$ ) (low quality of evidence). Furthermore, an additional RCT $(n=35)$ showed small effect and significant betweengroup difference on HOOS-sport at 6 weeks follow-up in favour of prescribed physiotherapy for hip joint pain (mean difference: 9.4 points, $95 \% \mathrm{CI} 0.1$ to 18.8 , Hedges $\mathrm{g}=0.46$ ) (very low quality of evidence). ${ }^{73}$

Comparison between different physiotherapy interventions

Three RCTs have compared different forms of physiotherapy interventions. ${ }^{227274}$ Aoyama et $\mathrm{al}^{74}(\mathrm{n}=24)$ showed a large and 
Table 3 Treatment of femoroacetabular impingement syndrome/labral injury: effect and grading the quality of evidence

\begin{tabular}{|c|c|c|c|c|c|}
\hline \multirow{2}{*}{$\begin{array}{l}\text { Between-group difference } \\
(95 \% \mathrm{Cl})\end{array}$} & & \multicolumn{4}{|l|}{ Effect size } \\
\hline & & Large & Medium & Small & Trivial \\
\hline \multicolumn{6}{|c|}{ Prescribed physiotherapy vs operative treatment } \\
\hline \multicolumn{6}{|c|}{ Meta-analysis } \\
\hline $\begin{array}{l}\text { iHOT-33 at } 8-12 \text { months follow-up; } \\
n=574^{20}\end{array}$ & $\begin{array}{l}11.02 \text { points }(4.83 ; 17.21) \text { in } \\
\text { favour of surgery, } I^{2}=43 \% \text {, } \\
\text { Hedges } g=0.41\end{array}$ & & & $\begin{array}{l}\text { Moderate quality of } \\
\text { evidence }\end{array}$ & \\
\hline \multicolumn{6}{|l|}{ Randomised controlled trial } \\
\hline $\begin{array}{l}\text { iHOT-33 at } 24 \text { months follow-up; } \\
\mathrm{n}=80^{70}\end{array}$ & $\begin{array}{l}6.3 \text { points }(-6.1 ; 18.7) \text { in } \\
\text { favour of surgery, Hedges } \\
g=0.23\end{array}$ & & & Low quality of evidence & \\
\hline $\begin{array}{l}\text { iHOT-33 at } 12 \text { months follow-up; } \\
\mathrm{n}=90^{81}\end{array}$ & $\begin{array}{l}12.11 \text { points }(3.27 ; 20.96) \\
\text { in favour of surgery, Hedges } \\
g=0.61\end{array}$ & & $\begin{array}{l}\text { Moderate quality of } \\
\text { evidence }\end{array}$ & & \\
\hline \multicolumn{6}{|c|}{$\begin{array}{l}\text { Prescribed physiotherapy vs passive modalities, stretching } \\
\text { and/or advice }\end{array}$} \\
\hline \multicolumn{6}{|c|}{ Meta-analysis } \\
\hline $\begin{array}{l}\text { IHOT-33 and NAHS at } 12 \text { weeks } \\
\text { follow-up; } n=54^{14}\end{array}$ & $\begin{array}{l}\text { Hedges } g=0.66(0.09 ; 1.23) \\
\text { in favour of prescribed } \\
\text { physiotherapy, } I^{2}=0 \%\end{array}$ & & Low quality of evidence & & \\
\hline \multicolumn{6}{|l|}{ Randomised controlled trial } \\
\hline $\begin{array}{l}\text { HOOS-Sport at } 6 \text { weeks follow-up } \\
\text { at; } n=35^{73}\end{array}$ & $\begin{array}{l}9.4 \text { points }(0.1 ; 18.8) \text { in favour } \\
\text { of prescribed physiotherapy, } \\
\text { Hedges } g=0.46\end{array}$ & & & $\begin{array}{l}\text { Very low quality of } \\
\text { evidence }\end{array}$ & \\
\hline \multicolumn{6}{|c|}{ Comparison between different physiotherapy interventions } \\
\hline \multicolumn{6}{|c|}{ Randomised controlled trial } \\
\hline $\begin{array}{l}\text { iHOT-12 at } 8 \text { weeks follow-up; core } \\
\text { and hip exercises vs hip exercises; } \\
n=24^{74}\end{array}$ & $\begin{array}{l}25.7 \text { point }(11.44 ; 39.96) \\
\text { in favour of core and hip } \\
\text { exercises, } \\
\text { Hedges } g=1.14\end{array}$ & $\begin{array}{l}\text { Very low quality of } \\
\text { evidence }\end{array}$ & & & \\
\hline $\begin{array}{l}\text { HOOS-Sport at } 12 \text { weeks } \\
\text { follow-up; standard training vs } \\
\text { 'movement pattern' training; } \\
n=46^{22}\end{array}$ & $\begin{array}{l}3.69 \text { points }(-4.36 ; 11.74) \text { in } \\
\text { favour of standard training, } \\
\text { Hedges } g=0.19\end{array}$ & & & & Low quality of evidence \\
\hline $\begin{array}{l}\text { HOOS-Sport at } 52 \text { weeks } \\
\text { follow-up; standard training vs } \\
\text { 'movement pattern' training; } \\
\mathrm{n}=46^{80}\end{array}$ & $\begin{array}{l}9.70 \text { points }(-2.19 ; 21.59) \text { in } \\
\text { favour of 'movement pattern' } \\
\text { training, } \\
\text { Hedges } g=0.53\end{array}$ & & $\begin{array}{l}\text { Very low quality of } \\
\text { evidence }\end{array}$ & & \\
\hline $\begin{array}{l}\text { HOS-Sport at } 6 \text { weeks follow- } \\
\text { up; hip exercises at home vs } \\
\text { manual therapy and supervised } \\
\text { physiotherapy; } n=18^{72}\end{array}$ & $\begin{array}{l}21.1 \text { points }(-9.1 ; 51.3) \text { in } \\
\text { favour of hip exercises, Hedges } \\
g=1.27\end{array}$ & $\begin{array}{l}\text { Very low quality of } \\
\text { evidence }\end{array}$ & & & \\
\hline \multicolumn{6}{|c|}{ Preoperative physiotherapy vs massage therapy } \\
\hline \multicolumn{6}{|c|}{ Randomised controlled trial } \\
\hline $\begin{array}{l}\text { NAHS at } 12 \text { weeks follow-up after } \\
\text { surgery; } n=18^{79}\end{array}$ & $\begin{array}{l}\text { No difference; raw values not } \\
\text { reported }\end{array}$ & & & & $\begin{array}{l}\text { Very low quality of } \\
\text { evidence }\end{array}$ \\
\hline
\end{tabular}

Prescribed postoperative physiotherapy vs advice

Meta-analysis

$\begin{array}{ll}\text { iHOT-33 at } 12-14 \text { weeks follow- } & 14.37 \text { points }(2.98 ; 25.77) \\ & \text { in favour of prescribed } \\ & \text { physiotherapy, } \\ & I^{2}=0 \% \text {, Hedges } g=0.67\end{array}$

\section{Randomised controlled trial}

\begin{tabular}{ll}
$\begin{array}{l}\text { iHOT-33 at } 24 \text { weeks follow-up; } \\
\mathrm{n}=28^{78}\end{array}$ & $\begin{array}{l}7.1 \text { points }(-5.5 ; 19.6) \\
\text { in favour of prescribed } \\
\text { physiotherapy, } \\
\text { Hedges } \mathrm{g}=0.38\end{array}$ \\
\hline $\mathrm{I}^{2}$ (heterogeneity in study results); Hedges $g$ assessed as trivial $(\mathrm{g}<0.2)$, small $(\mathrm{g} \geq 0.2)$, medium $(\mathrm{g} \geq 0.5)$ and large $(\mathrm{g} \geq 0.8)$. \\
HOOS, Hip Osteoarthritis Outcome Score; HOS, Hip Outcome Score; IHOT, International Hip Outcome Tool; NAHS, Non-Arthritic Hip Score.
\end{tabular}

significant between-group difference on iHOT-12 at 8-week follow-up in favour of hip and core exercises versus hip exercises alone for FAI syndrome (mean difference: 25 points, 95\% CI 11.44 to 39.96 , Hedges $g=1.14$ ) (very low quality of evidence).
Harris-Hayes et al $^{22}(\mathrm{n}=46)$ showed a trivial and non-significant between-group difference on HOOS-Sport at 12-week follow-up in favour of hip strengthening exercises versus movement pattern training for hip-related pain (mean difference: 3.69 
points, $95 \% \mathrm{CI}-4.36$ to 11.74 , Hedges $\mathrm{g}=0.19$ ) (low quality of evidence). In addition, 12 months follow-up of the same cohort showed a medium and non-significant between-group difference in favour of movement pattern training (mean difference: 9.70 points, $95 \% \mathrm{CI}-2.19$ to 21,59 , Hedges $\mathrm{g}=0.59$ ) (very low quality of evidence) ${ }^{80}$ Wright et $a l^{72}(\mathrm{n}=18)$ showed a large but non-significant between-group difference on HOS-Sport at 6-week follow-up in favour of hip exercises performed at home versus manual therapy and supervised physiotherapy for FAI syndrome (mean difference: 21.1 points, $95 \% \mathrm{CI}-9.1$ to 51.3 , Hedges $g=1.27$ ) (very low quality of evidence).

\section{Preoperative physiotherapy versus massage therapy}

One RCT $(n=18)^{79}$ showed a non-significant effect of 8 -week pre-operative physiotherapy versus massage on self-reported function, measured with NAHS, at 12 weeks post-surgery (mean difference not reported; very low quality of evidence).

\section{Prescribed postoperative physiotherapy versus advice}

A systematic review and meta-analysis, based on two RCTs $(\mathrm{n}=47),{ }^{7678}$ performed by Kemp et $a l^{14}$ showed a medium and significant between-group difference on iHOT-33 at 12-14 weeks follow-up in favour of prescribed postoperative physiotherapy for FAI syndrome ${ }^{78}$ and hip-related pain $^{76}$ (mean difference: 14.37 points, $95 \%$ CI 2.98 to $25.77, \mathrm{I}^{2}=0 \%$, Hedges $\mathrm{g}=0.67$ ) (low quality of evidence). Furthermore, one RCT $(n=28)$ also reported on 24 weeks follow-up, observing a small and non-significant between-group difference on iHOT-33 in favour of prescribed postoperative physiotherapy (mean difference: 7.1 points, 95\% CI -5.5 to 19.6 , Hedges $\mathrm{g}=0.38$ ) (low quality of evidence). ${ }^{78}$

\section{DISCUSSION}

In this statement paper, we have summarised the best available evidence and graded the quality of evidence concerning diagnosis (eg, special tests, self-reported symptoms, etc) and non-operative treatment for FAI syndrome/labral injuries. This statement paper extends on previous systematic reviews concerning diagnosis ${ }^{13151984-86}$ and treatment ${ }^{142087-93}$ by providing an updated comprehensive overview of diagnostic effectiveness for both clinical tests and self-reported patient history characteristics. Additionally, we used contemporary risk of bias assessments (Risk of Bias version 2.0 $)^{17}$ of RCTs. Thus, this statement provides updated clinical guidance for clinicians working with hip and groin pain patients and, based on the grading of the certainty of evidence, a foundation for clinical recommendations. ${ }^{16}$ In summary, only a few diagnostic tests seem able to assist in ruling FAI syndrome/labral injury in or out, prescribed physiotherapy seems to be the most effective non-operative treatment for FAI syndrome; however, based on current evidence, is inferior to hip arthroscopy.

\section{Diagnosis and clinical information}

Diagnosis of FAI syndrome/labral injuries remains a clinical challenge, ${ }^{29}$ possibly due to extra-articular causes of groin pain having a similar clinical presentation. ${ }^{95}$ The Warwick Agreement defined FAI syndrome to be present based on a combination of symptoms (eg, stiffness, pain, etc), clinical signs (eg, positive impingement test, restricted range of motion, etc), and radiological findings (cam and/or pincer morphology). ${ }^{1}$ For clinicians without easy access to imaging modalities, knowing the diagnostic effectiveness of specific symptoms and clinical signs for FAI syndrome/labral injury is useful.

We found 23 clinical tests and 14 self-reported patient history characteristics (clinical information); many of which provided very limited utility in clinical practice when the goal is to accurately diagnose FAI syndrome/labral injuries. On its own, clinical information was not useful for the diagnosis of FAI syndrome/labral injury. The most useful clinical test for ruling in FAI syndrome was prone restricted internal hip rotation in $0^{\circ}$ hip flexion (with knee in $90^{\circ}$ flexion) with or without pain. However, due to the combination of low quality of evidence and low diagnostic effectiveness, the interpretation of a positive test is that it may only slightly improve post-test probability. ${ }^{36}$ Nonetheless, the test showed high specificity of $94 \%,{ }^{45}$ indicating a low false-positive rate, ${ }^{96}$ and an LR + of 4.83 associated with a potential clinically relevant shift in pretest to post-test probability from 51\% (tertiary care setting $)^{45}$ to $83 \%$ following a positive test. However, it should be noted that the pretest probability is considerably lower in primary care ${ }^{97}$ or sports setting, ${ }^{98}$ also lowering the post-test probability. Therefore, a positive test in a primary care or sport setting is probably not sufficient to confirm the diagnosis of FAI syndrome. Furthermore, restricted internal hip rotation was based on a subjective assessment making it prone to misinterpretation, which is also reflected by a weak level of agreement between testers (kappa value: 0.43). ${ }^{45} 99$ Finally, while the reference standard to label FAI syndrome in the study included combined groin pain, cam/pincer morphology and $\geq 50 \%$ pain reduction during an ultrasound-guided hip injection, and thus closely resemble the Warwick Agreement, the optimal cut-point or definition of pain reduction after an injection to define intra-articular hip pain is uncertain. However, while pain reduction $>50 \%$ has been associated with cartilage injury ${ }^{100}$ which is often present in patients with FAI syndrome, ${ }^{9}$ this seems to be uncertain for labral injuries. ${ }^{50} 100$ These findings highlight the possibility that early-stage cases without cartilage injury but FAI syndrome may have been missed by the definition. The usefulness of restricted internal rotation for the diagnosis of FAI syndrome is partly in line with a Delphi study on diagnosis for FAI syndrome. Restricted internal rotation with pain (either with or without combined hip flexion) obtained consensus as a helpful component to include in the diagnostic process, whereas restricted internal rotation without pain did not. ${ }^{21}$

The tests with the best diagnostic effectiveness for ruling out FAI syndrome were no pain during FADIR and no restricted range of motion during FABER compared with the unaffected side. However, large heterogeneity in diagnostic effectiveness was observed between studies with negative LR - ranging from 0.09 to 0.83 (FADIR test) and $0.41-0.93$ (FABER test), making the clinical application uncertain. Furthermore, the quality of evidence was rated very low for the FADIR test suggesting that the test may either increase/decrease/or have no effect on the post-test probability. ${ }^{36}$ The quality of evidence was rated moderate for the FABER test and combined with a trivial to small diagnostic effectiveness, this suggests that at best the test probably decreases post-test probability slightly. ${ }^{36}$ However, the restricted range of motion in the FABER test was determined as a longer distance between the lateral aspect of the knee and the examination table, and based on a comparison with the unaffected hip without cam or pincer morphology. This requires the unaffected hip to undergo radiological examination for the test to be valid, and thus the clinical implication is questionable. ${ }^{54}$ This is supported by a Delphi study that 
failed to reach consensus on the usefulness of FABER test for diagnosis of FAI syndrome. ${ }^{21}$ The FADIR test has recently been highlighted in the International Hip-related Pain Research Network consensus statement on diagnosis of hip-related pain as a useful test to rule out FAI syndrome ${ }^{5}$ due to the test being very sensitive. ${ }^{95101}$ Since the test elicits high acetabular labral strains, ${ }^{102}$ and thus is expected to capture intra-articular pathology, no pain during the FADIR test is considered to rule out hip-related pain. Conversely, the test demonstrates poor specificity, ${ }^{95}$ representing a high false-positive rate. ${ }^{96}$ Therefore, using the FADIR test as an isolated confirmatory test to diagnose FAI syndrome/labral injury or hip-related pain is not recommended. ${ }^{521}$

Few clinical and self-reported tests were useful for diagnosis of labral injury ('clicking', FADIR test and Third-test), however, all were deemed to be of very low quality of evidence. Thus the effect estimate can be interpreted as very uncertain, indicating these tests may either increase/decrease/or lead to no change in the post-test probability. ${ }^{36}$

An inherent limitation of most diagnostic studies is the use of hip arthroscopy and/or imaging as the reference standard to diagnose FAI syndrome and/or labral injury. ${ }^{13}$ Given the high prevalence of cam and/or pincer morphology ${ }^{103}$ and labral injury in asymptomatic cases, ${ }^{104}$ morphological variations and imaging or arthroscopic findings may not always be the cause of pain, ${ }^{100} 105$ despite the labrum being densely populated by free nerve endings capable of transmitting nociception. ${ }^{106} 107$ Thus, the poor correlation seems to exist between hip joint morphology and pain ${ }^{100} 108$ and labral injury in symptomatic subjects undergoing hip arthroscopy is also poorly correlated to pain-relief after an intra-articular hip-joint anaesthetic injection. $^{50100}$

One of the cornerstones in diagnostic testing is to influence the choice of treatment approach and/or serve as a prognosis, with the aim of providing better outcomes for patients. ${ }^{37}$ All tests were downgraded due to indirectness. ${ }^{109}$ This is because it is currently unclear whether a specific diagnosis of hip-related pain $^{5}$ actually changes prognosis and/or initial management strategy for patients, which in most cases comprises exercisebased interventions. ${ }^{12} 110$

\section{Treatment}

A recent consensus statement on treatment for FAI syndrome advocated a minimum of 12 weeks of physiotherapist-led treatment focusing on hip muscle strengthening and functional performance as the initial approach before surgery is considered. ${ }^{12}$ Our findings also support the use of $6-12$ weeks of physiotherapist-led treatment (hip strengthening, manual therapy, functional training, movement pattern training) compared with passive modalities, stretching and/or advice (small to medium effect size). ${ }^{14}$ However, these findings are associated with low to very low quality of evidence, suggesting that at best prescribed physiotherapy may improve outcomes. The large uncertainty is primarily driven by high risk of bias, wide CIs, and use of inappropriate patient-reported outcome measure (NAHS and HOOS-Sport) ${ }^{26}$ in three studies. ${ }^{73} 7780$ Importantly, treatment outcomes after physiotherapist-led treatment may provide better results when patients are recruited through advertisements $^{75}$ versus an orthopaedic practice, ${ }^{69}{ }^{71}$ potentially reflecting patient bias regarding surgical treatment or differing disease severity status.

Three small RCTs with a 6-12 weeks follow-up compared different physiotherapy interventions. One study showed a large and significant effect of adding core exercises to a hip exercise programme, but the evidence is very uncertain due to the very low quality of evidence ${ }^{74}$; one study showed a large and non-significant effect of advice and home-based exercises versus manual therapy and supervised physiotherapy, but the evidence is very uncertain due to the very low level of evidence ${ }^{72}$; one study showed a trivial and nonsignificant difference between movement-pattern training and standard rehabilitation suggesting that the interventions may result in no difference (low level of evidence). ${ }^{22}$ However, 12-month follow-up suggests that within-group improvements are retained, indicating a potential long-term effect of nonoperative treatment outcome. ${ }^{80}$ Due to the heterogeneity of physiotherapy interventions between these studies, it seems difficult to recommend a specific non-operative treatment approach (eg, movement pattern training vs hip strengthening) beyond exercise-based treatment. ${ }^{12}$ The mechanisms of improvements following exercise-based treatment are yet to be elucidated, but may be related to improvements in hip muscle strength ${ }^{14}$ and altered hip joint kinematics (ie, reduced hip adduction angle during single leg squatting) ${ }^{111}$ potentially reflecting better load-bearing capacity of the hip joint. ${ }^{112} 113$

In individuals eligible for surgery, a meta-analysis of three RCTs $^{69-71}$ showed a small effect size in favour of hip arthroscopy for improving hip-related quality of life (iHOT-33) compared with prescribed physiotherapy at a follow-up of 8-12 months suggesting that hip arthroscopy probably improves iHOT-33 slightly more (moderate quality of evidence). ${ }^{20}$ However, the prescribed physiotherapy intervention was poorly described in all studies potentially limiting real-world implementation, ${ }^{69-71}$ and may not represent contemporary physiotherapist-led treatment. ${ }^{114}$ Noteworthy, the $95 \%$ CIs ranged from 4.83 to 17.21 points (iHOT-33), where the lower end does not exceed the minimal clinically important difference of 6 points, ${ }^{28}$ indicating that future studies may alter the conclusion. Prescribed postoperative physiotherapy including exercises and manual therapy versus advice showed a medium effect size for improving self-reported hip function after surgery for FAI syndrome and hip-pain indicating that this may increase postoperative outcomes (low quality of evidence). ${ }^{76}$ This is in line with a survey on postoperative practices among surgeons and physiotherapists, where $>85 \%$ rated exercise therapy as 'very important' or 'extremely important'. ${ }^{115}$

Although both operative treatment and prescribed physiotherapy are associated with improvements in self-reported function, many patients still report problems following both treatments, as indicated by the proportion not obtaining an acceptable symptom state following either surgery $(50 \%)^{71} 116$ or prescribed physiotherapy (up to $63 \%-81 \%$ ). ${ }^{71}{ }^{72}$ This also seems to be the case regarding sports participation, with many athletes being unable to reach their preinjury level of sport and performance after treatment. ${ }^{311-121}$ Notably, 25\% of physiotherapists and 50\% of surgeons reported in a survey that they did not evaluate the readiness to sport after surgery and postoperative rehabilitation, which may leave many patients on their own in terms of managing the transition back to the sport. $^{115}$

\section{Methodological considerations}

The current statement has potential methodological limitations. We decided a priori only to include RCTs on treatment, although prospective cohort studies on treatment outcomes of non-operative treatment for FAI/labral injury have been 
What is already known?

- Femoroacetabular impingement (FAI) syndrome/labral injuries is a recognised cause of hip-related groin pain.

- A comprehensive overview with grading of the quality of evidence related to diagnosis and non-operative treatment is lacking.

\section{What are the new findings?}

- Restricted internal hip rotation in $0^{\circ}$ hip flexion with or without pain was the best test to rule in FAl syndrome, however, the diagnostic effectiveness and quality of evidence was low, indicating high uncertainty in the estimate, and practically the assessment was prone to misinterpretation.

- No pain in Flexion Adduction Internal Rotation test and no restricted range of motion in Flexion Abduction External Rotation test compared with the unaffected side were best to rule out FAI syndrome.

- No forms of clinical information, such as self-reported pain location, clicking, locking, giving way were useful for diagnosis of FAI syndrome/labral injury.

- Prescribed physiotherapy, consisting of hip strengthening, hip joint manual therapy techniques, functional activityspecific retraining, and education may be slightly superior to passive modalities, but are probably slightly inferior to hip arthroscopy.

- Most outcomes were graded as very low to moderate quality of evidence with wide $\mathrm{Cls}$, thus further high-quality research is likely to have an important impact on the confidence of these findings and recommendations.

published. ${ }^{118}$ 122-126 This was chosen since RCTs represent the highest starting point for the GRADE assessment, ${ }^{16}$ although low risk of bias cohort studies may yield an equal quality of evidence as a high risk of bias RCT. For a systematic review including cohort studies on treatment for hip pain, we refer to Kemp et al. ${ }^{14}$ In addition, we did not include treatment studies focusing solely on therapeutic hip injection, although we appreciate such modalities constitute non-operative treatment and is included in the Warwick Agreement as a treatment option. This was an a priori decision since sports physical therapists in Denmark are not allowed to practice injection therapy. ${ }^{1}$ Few studies have been published on therapeutic hip injections as non-operative treatment in FAI syndrome, showing small decrements in shortterm hip pain ( $<2$ months) and improvements in long-term (12 months) self-reported hip function; however, none of the studies included a control group. ${ }^{93} 127$ Since treatment studies normally use several outcome measures such as self-reported measures, muscle strength, biomechanical analyses, we decided a priori only to include data on self-reported measures. This was chosen in accordance with the GRADE framework, as patientreported outcome measures represent patient-centred outcomes and thus contain the lowest risk of downgrading due to indirectness. ${ }^{33}$ The inclusion of three databases for the literature search (Medline, CENTRAL and Embase) may be perceived as a limitation. However, for musculoskeletal disorders these databases cover most literature, with a potential of missing only $2 \%,{ }^{24}$ and is recommended by the Methodological Expectations of Cochrane Intervention Reviews standards as the minimum databases to be covered. ${ }^{128}$ To increase the likelihood of identifying potential additional studies not covered by our literature search, we used alternative ways of identifying relevant literature such as checking reference lists of all systematic reviews identified. ${ }^{128}$

\section{CONCLUSION}

For diagnostic tests, restricted internal hip rotation in $0^{\circ}$ hip flexion with or without pain was the best test to rule in FAI syndrome (low diagnostic effectiveness; low quality of evidence; interpretation of evidence: may increase post-test probability slightly), whereas no pain in FADIR test and no restricted range of motion in FABER test were best to rule out (very low to high diagnostic effectiveness; very low to moderate quality of evidence; interpretation of evidence: very uncertain, but may reduce post-test probability slightly). Clinical information such as self-reported symptoms was not useful for diagnosis. For treatment, prescribed physiotherapy consisting of hip strengthening, hip joint manual therapy techniques, functional activity-specific retraining and education showed a small to medium effect size compared with passive modalities, stretching and/or advice (very low to low quality of evidence; interpretation of evidence: very uncertain, but may slightly improve outcomes); however, prescribed physiotherapy was inferior to hip arthroscopy (small effect size; moderate quality of evidence; interpretation of evidence: hip arthroscopy probably improves outcomes slightly). For both domains, the overall quality of evidence ranged from very low to moderate. All treatment comparisons were associated with wide CIs, often crossing the line for minimal clinically important difference, indicating that future research on diagnosis and treatment may alter the conclusions from this review.

\section{Author affiliations}

${ }^{1}$ Sports Orthopaedic Research Center-Copenhagen (SORC-C), Arthroscopic Center, Department of Orthopedic Surgery, Copenhagen University Hospital, Hvidovre Hospital, Hvidovre, Denmark

${ }^{2}$ Physical Medicine \& Rehabilitation Research - Copenhagen (PMR-C), Department of Orthopedic Surgery and Physical Therapy, Copenhagen University Hospital, Hvidovre, Denmark

${ }^{3}$ Department of Clinical Research, Copenhagen University Hospital Hvidovre, Hvidovre, Denmark

${ }^{4}$ Danish Society of Sports Physical Therapy, Odense, Denmark

Twitter Lasse Ishøi @Lasselshoei, Mathias Fabricius Nielsen @Physiomathias, Kasper Krommes @krommes, Rasmus Skov Husted @Husted_RS and Kristian Thorborg@KThorborg

Acknowledgements We would like to acknowledge the Danish Society of Sports Physical Therapy (DSSF) for supporting this project.

Contributors $\mathrm{LI}$ and $\mathrm{KT}$ conceived the study idea. KK performed the systematic searches with input from LI, MFN, KT, RSH, LLP. LI, MFN and RSH conducted risk of bias assessments, while LI and MFN conducted grade assessments. LI wrote the initial draft. All authors revised the draft critically and agreed on the final version.

Funding The Danish Society of Sports Physical Therapy initiated the project and provided financial support to authors (LI, RSH, LLP, KT).

Competing interests $\mathrm{KT}$ is Deputy Editor in BJSM, and have received grants from the Danish Society of Sports Physical Therapy.

Patient consent for publication Not required.

Provenance and peer review Not commissioned; externally peer reviewed.

Supplemental material This content has been supplied by the author(s). It has not been vetted by BMJ Publishing Group Limited (BMJ) and may not have been peer-reviewed. Any opinions or recommendations discussed are solely those of the author(s) and are not endorsed by BMJ. BMJ disclaims all liability and responsibility arising from any reliance placed on the content. Where the content includes any translated material, BMJ does not warrant the accuracy and reliability of the translations (including but not limited to local regulations, clinical guidelines, terminology, drug names and drug dosages), and is not responsible for any error and/or omissions arising from translation and adaptation or otherwise. 
ORCID iDs

Lasse Ishøi http://orcid.org/0000-0002-2716-6567

Mathias Fabricius Nielsen http://orcid.org/0000-0003-4211-3516

Kasper Krommes http://orcid.org/0000-0001-9856-7547

Rasmus Skov Husted http://orcid.org/0000-0003-2020-8792

Per Hölmich http://orcid.org/0000-0003-2098-0272

Lisbeth Lund Pedersen http://orcid.org/0000-0002-3283-622X

Kristian Thorborg http://orcid.org/0000-0001-9102-4515

\section{REFERENCES}

1 Griffin DR, Dickenson EJ, O'Donnell J, et al. The Warwick agreement on femoroacetabular impingement syndrome (FAl syndrome): an international consensus statement. Br J Sports Med 2016;50:1169-76.

2 Clohisy JC, Baca G, Beaulé PE, et al. Descriptive epidemiology of femoroacetabular impingement: a North American cohort of patients undergoing surgery. Am J Sports Med 2013;41:1348-56

3 Ishøi L, Thorborg K, Kraemer O, et al. Return to sport and performance after hip arthroscopy for femoroacetabular impingement in 18- to 30-year-old athletes: a cross-sectional cohort study of 189 athletes. Am J Sports Med 2018:46:2578-87.

4 Thorborg K, Kraemer O, Madsen A-D, et al. Patient-Reported outcomes within the first year after hip arthroscopy and rehabilitation for femoroacetabular impingement and/or Labral injury: the difference between getting better and getting back to normal. Am J Sports Med 2018;46:2607-14.

5 Reiman MP, Agricola R, Kemp JL. Consensus recommendations on the classification, definition and diagnostic criteria of hip-related pain in young and middle-aged active adults from the International Hip-related pain research network, Zurich 2018 Br J Sports Med (Published Online First: 20 January 2020).

6 Nogier A, Bonin N, May 0, et al. Descriptive epidemiology of mechanical hip pathology in adults under 50 years of age. prospective series of 292 cases: clinical and radiological aspects and physiopathological review. Orthop Traumato/ Surg Res 2010;96:\$53-8.

7 de Sa D, Hölmich P, Phillips M, et al. Athletic groin pain: a systematic review of surgical diagnoses, investigations and treatment. Br J Sports Med 2016;50:1181-6.

8 Kemp JL, Makdissi M, Schache AG, et al. Hip chondropathy at arthroscopy: prevalence and relationship to labral pathology, femoroacetabular impingement and patient-reported outcomes. Br J Sports Med 2014;48:1102-7.

9 Ishøi L, Thorborg K, Kraemer O, et al. Demographic and radiographic factors associated with intra-articular hip cartilage injury: a cross-sectional study of 1511 hip arthroscopy procedures. Am J Sports Med 2019;47:2617-25.

10 Liu Q, Wang W, Thoreson AR, et al. Finite element prediction of contact pressures in cam-type femoroacetabular impingement with varied alpha angles. Comput Methods Biomech Biomed Engin 2017;20:294-301.

11 Wyles CC, Norambuena GA, Howe BM, et al. Cam deformities and limited hip range of motion are associated with early osteoarthritic changes in adolescent athletes: a prospective matched cohort study. Am J Sports Med 2017;45:3036-43.

12 Kemp JL, Risberg MA, Mosler A, et al. Physiotherapist-led treatment for young to middle-aged active adults with hip-related pain: consensus recommendations from the International Hip-related pain research network, Zurich 2018. Br J Sports Med 2020;54:504-11

13 Reiman MP, Goode AP, Cook CE, et al. Diagnostic accuracy of clinical tests for the diagnosis of hip femoroacetabular impingement/labral tear: a systematic review with meta-analysis. Br J Sports Med 2015;49:811.

14 Kemp JL, Mosler AB, Hart H, et al. Improving function in people with hip-related pain: a systematic review and meta-analysis of physiotherapist-led interventions for hip-related pain. Br J Sports Med 2020;54:1382-94.

15 Burgess RM, Rushton A, Wright C, et al. The validity and accuracy of clinical diagnostic tests used to detect labral pathology of the hip: a systematic review. Man Ther 2011;16:318-26.

16 Schünemann H, Brożek J, Guyatt G. Handbook for grading the quality of evidence and the strength of recommendations using the grade approach, 2013.

17 Sterne JAC, Savović J, Page MJ, et al. Rob 2: a revised tool for assessing risk of bias in randomised trials. BMJ 2019;366:14898.

18 Whiting PF, Rutjes AWS, Westwood ME, et al. QUADAS-2: a revised tool for the quality assessment of diagnostic accuracy studies. Ann Intern Med 2011;155:529-36.

19 Caliesch R, Sattelmayer M, Reichenbach S, et al. Diagnostic accuracy of clinical tests for CaM or pincer morphology in individuals with suspected FAl syndrome: a systematic review. BMJ Open Sport Exerc Med 2020;6:e000772.

20 Ferreira GE, O'Keeffe M, Maher CG, et al. The effectiveness of hip arthroscopic surgery for the treatment of femoroacetabular impingement syndrome: a systematic review and meta-analysis. J Sci Med Sport 2021;24:21-9.

21 Reiman MP, Thorborg K, Covington K, et al. Important clinical descriptors to include in the examination and assessment of patients with femoroacetabular impingement syndrome: an international and multi-disciplinary Delphi survey. Knee Surg Sports Traumatol Arthrosc 2017;25:1975-1986.

22 Harris-Hayes M, Steger-May K, Bove AM, et al. Movement pattern training compared with standard strengthening and flexibility among patients with hip-related groin pain: results of a pilot multicentre randomised clinical trial. BMJ Open Sport Exerc Med 2020:6:e000707.

23 Howick J, Chalmers I, Glasziou P. Explanation of the 2011 Oxford centre for evidence-based medicine (OCEBM) levels of evidence (background document. Oxford Centre for Evidence-Based Medicine.

24 Aagaard T, Lund $\mathrm{H}$, Juhl C. Optimizing literature search in systematic reviews - are MEDLINE, EMBASE and CENTRAL enough for identifying effect studies within the area of musculoskeletal disorders? BMC Med Res Methodol 2016;16:161.

25 Ishøi L, Krommes K, Husted RS, et al. Diagnosis, prevention and treatment of common lower extremity muscle injuries in sport - grading the evidence: a statement paper commissioned by the Danish Society of Sports Physical Therapy (DSSF). Br J Sports Med 2020;54:528-37.

26 Impellizzeri FM, Jones DM, Griffin D, et al. Patient-Reported outcome measures for hip-related pain: a review of the available evidence and a consensus statement from the International Hip-related pain research network, Zurich 2018. Br J Sports Med 2020;54:848-57.

27 Thorborg K, Hölmich P, Christensen R, et al. The Copenhagen hip and groin outcome score (HAGOS): development and validation according to the COSMIN checklist. $\mathrm{Br} J$ Sports Med 2011;45:478-91.

28 Mohtadi NGH, Griffin DR, Pedersen ME, et al. The development and validation of a self-administered quality-of-life outcome measure for young, active patients with symptomatic hip disease: the International hip outcome tool (iHOT-33). Arthroscopy 2012:28:595-610.

29 Guyatt GH, Oxman AD, Vist G, et al. GRADE guidelines: 4. Rating the quality of evidence--study limitations (risk of bias). J Clin Epidemio/ 2011;64:407-15.

30 ROBIS: A new tool to assess risk of bias in systematic reviews was developed. - PubMed - NCBI. Available: https://www.ncbi.nlm.nih.gov/pubmed/26092286 [Accessed 12 Sep 2019].

31 Cochrane Handbook for systematic reviews of interventions. Available: https:// handbook-5-1.cochrane.org/index.htm\#chapter_9/9_5_heterogeneity.htm [Accessed 14 Sep 2019].

32 Guyatt GH, Oxman AD, Kunz R, et al. GRADE guidelines: 7. Rating the quality of evidence--inconsistency. J Clin Epidemiol 2011;64:1294-302.

33 Guyatt GH, Oxman AD, Kunz R, et al. GRADE guidelines: 8. Rating the quality of evidence--indirectness. J Clin Epidemiol 2011;64:1303-10.

34 Guyatt GH, Oxman AD, Kunz R, et al. GRADE quidelines 6 . Rating the quality of evidence--imprecision. J Clin Epidemiol 2011;64:1283-93.

35 Guyatt GH, Oxman AD, Montori V, et al. GRADE guidelines: 5 . Rating the quality of evidence--publication bias. J Clin Epidemio/ 2011;64:1277-82.

36 Santesso N, Glenton C, Dahm P, et al. Grade guidelines 26: informative statements to communicate the findings of systematic reviews of interventions. J Clin Epidemiol 2020;119:126-35.

37 Jaeschke R, Guyatt GH, Sackett DL. Users' guides to the medical literature. III. How to use an article about a diagnostic test. B. what are the results and will they help me in caring for my patients? the evidence-based medicine Working group. JAMA 1994;271:703-7.

38 McGee S. Simplifying likelihood ratios. J Gen Intern Med 2002;17:647-50.

39 Chapter 6: choosing effect measures and computing estimates of effect. Available: . I handbook/current/chapter-06 [Accessed 12 Dec 2020].

40 Greenland S, Robins JM. Estimation of a common effect parameter from sparse follow-up data. Biometrics 1985;41:55-68.

41 Higgins JPT, Thompson SG, Deeks JJ, et al. Measuring inconsistency in meta-analyses. BMJ 2003:327:557-60.

42 Cohen J. A power primer. Psychol Bull 1992;112:155-9.

43 Hedges LV. Distribution Theory for Glass's Estimator of Effect size and Related Estimators. J Educ Stat 1981;6:107-28.

44 Tijssen $M$, van Cingel REH, de Visser E, et al. Hip joint pathology: relationship between patient history, physical tests, and arthroscopy findings in clinical practice. Scand J Med Sci Sports 2017:27:342-50.

45 Pålsson A, Kostogiannis I, Ageberg E. Combining results from hip impingement and range of motion tests can increase diagnostic accuracy in patients with FAl syndrome. Knee Surg Sports Traumatol Arthrosc 2020;28:3382-92.

46 Hananouchi T, Yasui Y Yamamoto K, et al. Anterior impingement test for labral lesions has high positive predictive value. Clin Orthop Relat Res 2012:470:3524-9.

47 Peters CL, Schabel K, Anderson L, et al. Open treatment of femoroacetabular impingement is associated with clinical improvement and low complication rate at short-term followup. Clin Orthop Relat Res 2010;468:504-10.

48 Domayer SE, Ziebarth K, Chan J, et al. Femoroacetabular cam-type impingement: diagnostic sensitivity and specificity of radiographic views compared to radial MRI Eur J Radiol 2011:80:805-10.

49 Sink EL, Gralla J, Ryba A, et al. Clinical presentation of femoroacetabular impingement in adolescents. J Pediatr Orthop 2008;28:806-11.

50 Martin RL, Irrgang JJ, Sekiya JK. The diagnostic accuracy of a clinical examination in determining intra-articular hip pain for potential hip arthroscopy candidates. Arthroscopy 2008;24:1013-8.

51 Ranawat AS, Gaudiani MA, Slullitel PA, et al. Foot progression angle walking test: a dynamic diagnostic assessment for femoroacetabular impingement and hip instability. Orthop J Sports Med 2017;5:2325967116679641. 
52 Owusu-Akyaw KA, Hutyra CA, Evanson RJ, et al. Concurrent validity of a patient self-administered examination and a clinical examination for femoroacetabular impingement syndrome. BMJ Open Sport Exerc Med 2019;5:e000574

53 Maslowski E, Sullivan W, Forster Harwood J, et al. The diagnostic validity of hip provocation maneuvers to detect intra-articular hip pathology. $P m R$ 2010;2:174-81.

54 Trindade CAC, Briggs KK, Fagotti L, et al. Positive FABER distance test is associated with higher alpha angle in symptomatic patients. Knee Surg Sports Traumatol Arthrosc 2019;27:3158-61.

55 Chan Y-S, Lien L-C, Hsu H-L, et al. Evaluating hip labral tears using magnetic resonance arthrography: a prospective study comparing hip arthroscopy and magnetic resonance arthrography diagnosis. Arthroscopy 2005:21:1250.

56 Troelsen A, Mechlenburg I, Gelineck J, et al. What is the role of clinical tests and ultrasound in acetabular labral tear diagnostics? Acta Orthop 2009;80:314-8.

57 Myrick KM, Nissen CW. Third test: diagnosing hip Labral tears with a new physical examination technique. J Nurse Pract 2013;9:501-5.

$58 \mathrm{McC}$ arthy JC, Busconi B. The role of hip arthroscopy in the diagnosis and treatment of hip disease. Orthopedics 1995;18:753-6.

59 Narvani AA, Tsiridis E, Kendall S, et al. A preliminary report on prevalence of acetabular labrum tears in sports patients with groin pain. Knee Surg Sports Traumatol Arthrosc 2003;11:403-8.

60 Laude F, Sariali E, Nogier A. Femoroacetabular impingement treatment using arthroscopy and anterior approach. Clin Orthop Relat Res 2009;467:747-52.

61 Leunig M, Werlen S, Ungersböck A, et al. Evaluation of the acetabular labrum by $\mathrm{Mr}$ arthrography. J Bone Joint Surg Br 1997;79:230-4.

62 Ayeni $\mathrm{O}, \mathrm{Chu} \mathrm{R}$, Hetaimish B, et al. A painful squat test provides limited diagnostic utility in CAM-type femoroacetabular impingement. Knee Surg Sports Traumatol Arthrosc 2014;22:806-11

63 Aprato A, Massè A, Faletti C, et al. Magnetic resonance arthrography for femoroacetabular impingement surgery: is it reliable? J Orthop Traumatol 2013;14:201-6.

64 Beaulé PE, Zaragoza E, Motamedi K, et al. Three-Dimensional computed tomography of the hip in the assessment of femoroacetabular impingement. J Orthop Res 2005;23:1286-92.

65 Keeney JA, Peelle MW, Jackson J, et al. Magnetic resonance arthrography versus arthroscopy in the evaluation of articular hip pathology. Clin Orthop Relat Res 2004:163-9.

66 Petersilge CA, Haque MA, Petersilge WJ, et al. Acetabular labral tears: evaluation with Mr arthrography. Radiology 1996;200:231-5.

67 Barton C, Salineros MJ, Rakhra KS, et al. Validity of the alpha angle measurement on plain radiographs in the evaluation of cam-type femoroacetabular impingement. Clin Orthop Relat Res 2011;469:464-9.

68 Wang W-guo, Yue D-bo, Zhang N-fei, et al. Clinical diagnosis and arthroscopic treatment of acetabular labral tears. Orthop Surg 2011;3:28-34.

69 Griffin DR, Dickenson EJ, Wall PDH, et al. Hip arthroscopy versus best conservative care for the treatment of femoroacetabular impingement syndrome (UK fashion): a multicentre randomised controlled trial. Lancet 2018;391:2225-35.

70 Mansell NS, Rhon DI, Meyer J, et al. Arthroscopic surgery or physical therapy for patients with femoroacetabular impingement syndrome: a randomized controlled trial with 2-year follow-up. Am J Sports Med 2018;46:1306-14.

71 Palmer AJR, Ayyar Gupta V, Fernquest S, et al. Arthroscopic hip surgery compared with physiotherapy and activity modification for the treatment of symptomatic femoroacetabular impingement: multicentre randomised controlled trial. BMJ 2019:364:1185.

72 Wright AA, Hegedus EJ, Taylor JB, et al. Non-Operative management of femoroacetabular impingement: a prospective, randomized controlled clinical trial pilot study. J Sci Med Sport 2016:19:716-21.

73 Harris-Hayes M, Czuppon S, Van Dillen LR, et al. Movement-Pattern training to improve function in people with chronic hip joint pain: a feasibility randomized clinical trial. J Orthop Sports Phys Ther 2016;46:452-61.

74 Aoyama M, Ohnishi Y, Utsunomiya $\mathrm{H}$, et al. A prospective, randomized, controlled trial comparing conservative treatment with trunk stabilization exercise to standard hip muscle exercise for treating femoroacetabular impingement: a pilot study. Clin J Sport Med 2019;29:267-75

75 Kemp JL, Coburn SL, Jones DM, et al. The physiotherapy for femoroacetabular impingement rehabilitation study (physioFIRST): a pilot randomized controlled trial. Orthop Sports Phys Ther 2018;48:307-15

76 Kemp J, Moore K, Fransen M, et al. A pilot randomised clinical trial of physiotherapy (manual therapy, exercise, and education) for early-onset hip osteoarthritis post-hip arthroscopy. Pilot Feasibility Stud 2018;4:16

77 Smeatham A, Powell R, Moore S, et al. Does treatment by a specialist physiotherapist change pain and function in young adults with symptoms from femoroacetabular impingement? A pilot project for a randomised controlled trial. Physiotherapy 2017:103:201-7.

78 Bennell KL, Spiers L, Takla A, et al. Efficacy of adding a physiotherapy rehabilitation programme to arthroscopic management of femoroacetabular impingement syndrome: a randomised controlled trial (fair). BMJ Open 2017;7:e014658.
79 Grant LF, Cooper DJ, Conroy JL. The HAPI 'Hip Arthroscopy Pre-habilitation Intervention' study: does pre-habilitation affect outcomes in patients undergoing hip arthroscopy for femoro-acetabular impingement? J Hip Preserv Surg 2017:4:85-92.

80 Harris-Hayes M, Steger-May K, M Bove A, et al. One-Year outcomes following physical therapist-led intervention for chronic hip-related groin pain: ancillary analysis of a pilot multicenter randomized clinical trial. J Orthop Res 2021 doi:10.1002/jor.24985. [Epub ahead of print: 17 Jan 2021].

81 Martin SD, Abraham PF, Varady NH, et al. Hip arthroscopy versus physical therapy for the treatment of symptomatic acetabular Labral tears in patients older than 40 years: a randomized controlled trial. Am J Sports Med 2021;49:1199-208.

$82 \mathrm{Kim}$ C-H, Moon J-K, Yoon JY, et al. Arthroscopy versus nonoperative treatment of symptomatic femoroacetabular impingement syndrome: a protocol for systematic review and meta-analysis. Medicine 2020;99:e23247.

83 Schwabe MT, Clohisy JC, Cheng AL, et al. Short-Term clinical outcomes of hip arthroscopy versus physical therapy in patients with femoroacetabular impingement: a systematic review and meta-analysis of randomized controlled trials. Orthop J Sports Med 2020;8:2325967120968490

84 Tijssen $M$, van Cingel R, Willemsen L, et al. Diagnostics of femoroacetabular impingement and labral pathology of the hip: a systematic review of the accuracy and validity of physical tests. Arthroscopy 2012;28:860-71.

85 Pacheco-Carrillo A, Medina-Porqueres I. Physical examination tests for the diagnosis of femoroacetabular impingement. A systematic review. Phys Ther Sport 2016;21:87-93

86 Shanmugaraj A, Shell JR, Horner NS, et al. How useful is the Flexion-AdductionInternal rotation test for diagnosing femoroacetabular impingement: a systematic review. Clin J Sport Med 2020;30:76-82

87 Casartelli NC, Valenzuela PL, Maffiuletti NA, et al. Effectiveness of hip arthroscopy on treatment of femoroacetabular impingement syndrome: a meta-analysis of randomized controlled trials. Arthritis Care Res 2021;73:1140-5.

88 Dwyer T, Whelan D, Shah PS, et al. Operative versus Nonoperative treatment of femoroacetabular impingement syndrome: a meta-analysis of short-term outcomes. Arthroscopy 2020;36:263-73

89 Gatz M, Driessen A, Eschweiler J, et al. Arthroscopic surgery versus physiotherapy for femoroacetabular impingement: a meta-analysis study. Eur J Orthop Surg Traumatol 2020;30:1151-62

90 Hoit G, Whelan DB, Dwyer T, et al. Physiotherapy as an initial treatment option for femoroacetabular impingement: a systematic review of the literature and metaanalysis of 5 randomized controlled trials. Am J Sports Med 2020:48:2042-50.

91 Wall PDH, Fernandez M, Griffin DR, et al. Nonoperative treatment for femoroacetabular impingement: a systematic review of the literature. $P m R$ 2013:5:418-26

92 Bastos RM, de Carvalho Júnior JG, da Silva SAM, et al. Surgery is no more effective than conservative treatment for femoroacetabular impingement syndrome: systematic review and meta-analysis of randomized controlled trials. Clin Rehabil 2021;35:332-41.

93 Mallets E, Turner A, Durbin J, et al. Short-Term outcomes of conservative treatment for femoroacetabular impingement: a systematic review and meta-analysis. Int J Sports Phys Ther 2019;14:514-24.

94 Burnett RSJ, Della Rocca GJ, Prather H, et al. Clinical presentation of patients with tears of the acetabular labrum. J Bone Joint Surg Am 2006;88:1448-57.

95 Pålsson A, Kostogiannis I, Lindvall H, et al. Hip-related groin pain, patient characteristics and patient-reported outcomes in patients referred to tertiary care due to longstanding hip and groin pain: a cross-sectional study. BMC Musculoskelet Disord 2019:20:432

96 Kent P, Hancock MJ. Interpretation of dichotomous outcomes: sensitivity, specificity, likelihood ratios, and pre-test and post-test probability. J Physiother 2016;62:231-3.

97 Röling MA, Mathijssen NMC, Bloem RM. Incidence of symptomatic femoroacetabular impingement in the general population: a prospective registration study. J Hip Preserv Surg 2016:3:203-7

98 Ekstrand J, Hägglund M, Waldén M. Injury incidence and injury patterns in professional football: the UEFA injury study. Br J Sports Med 2011;45:553-8.

99 McHugh ML. Interrater reliability: the kappa statistic. Biochem Med $2012 \cdot 22 \cdot 276-82$

100 Kivlan BR, Martin RL, Sekiya JK. Response to diagnostic injection in patients with femoroacetabular impingement, labral tears, chondral lesions, and extra-articula pathology. Arthroscopy 2011;27:619-27

101 Izumi M, Petersen KK, Arendt-Nielsen L, et al. Pain referral and regiona deep tissue hyperalgesia in experimental human hip pain models. Pain 2014:155:792-800

102 Safran MR, Giordano G, Lindsey DP, et al. Strains across the acetabular labrum during hip motion: a cadaveric model. Am J Sports Med 2011;39 Suppl:92S-102.

103 Mosler AB, Crossley KM, Waarsing JH, et al. Ethnic differences in bony hip morphology in a cohort of 445 professional male soccer players. Am J Sports Med 2016:44:2967-74.

104 Heerey JJ, Kemp JL, Mosler AB, et al. What is the prevalence of imaging-defined intra-articular hip pathologies in people with and without pain? A systematic review and meta-analysis. Br J Sports Med 2018;52:581-93. 
105 Arnold DR, Keene JS, Blankenbaker DG, et al. Hip pain referral patterns in patients with labral tears: analysis based on intra-articular anesthetic injections, hip arthroscopy, and a new pain "circle" diagram. Phys Sportsmed 2011;39:29-35.

106 Gerhardt M, Johnson K, Atkinson R, et al. Characterisation and classification of the neural anatomy in the human hip joint. Hip Int 2012;22:75-81.

107 Alzaharani A, Bali K, Gudena R, et al. The innervation of the human acetabular labrum and hip joint: an anatomic study. BMC Musculoskelet Disord 2014;15:41.

108 Kierkegaard S, Rømer L, Lund B, et al. No association between femoral or acetabular angles and patient-reported outcomes in patients with femoroacetabular impingement syndrome-results from the HAFAI cohort. J Hip Preserv Surg 2020;7:242-8.

109 Schünemann HJ, Schünemann AHJ, Oxman AD, et al. Grading quality of evidence and strength of recommendations for diagnostic tests and strategies. BMJ 2008;336:1106-10.

110 Thorborg K, Reiman MP, Weir A, et al. Clinical examination, diagnostic imaging, and testing of athletes with groin pain: an evidence-based approach to effective management. J Orthop Sports Phys Ther 2018;48:239-49.

111 Harris-Hayes M, Steger-May K, van Dillen LR, et al. Reduced hip adduction is associated with improved function after Movement-Pattern training in young people with chronic hip joint pain. J Orthop Sports Phys Ther 2018:48:316-24.

112 Samaan MA, Zhang AL, Popovic T, et al. Hip joint muscle forces during gait in patients with femoroacetabular impingement syndrome are associated with patient reported outcomes and cartilage composition. J Biomech 2019;84:138-46.

$113 \mathrm{Ng} \mathrm{KCG}$, Mantovani G, Modenese L, et al. Altered walking and muscle patterns reduce hip contact forces in individuals with symptomatic CaM femoroacetabular impingement. Am J Sports Med 2018;46:2615-23.

114 Kemp JL, King MG, Barton C, et al. Is exercise therapy for femoroacetabular impingement in or out of fashion? We need to talk about current best practice for the non-surgical management of FAl syndrome. Br J Sports Med 2019:53:1204-5.

115 Wörner T, Thorborg K, Moksnes H, et al. Similar views on rehabilitation following hip arthroscopy among physiotherapists and surgeons in Scandinavia: a specialized care survey. Knee Surg Sports Traumatol Arthrosc 2018;26:2519-26.

116 Ishøi L, Thorborg K, Ørum MG, et al. How many patients achieve an acceptable symptom state after hip arthroscopy for femoroacetabular impingement syndrome? A cross-sectional study including pass cutoff values for the HAGOS and iHOT-33. Orthop J Sports Med 2021;9:232596712199526.

117 Wörner T, Thorborg K, Stålman A, et al. High or low return to sport rates following hip arthroscopy is a matter of definition? Br J Sports Med 2018;52:1475-6.
118 Pennock AT, Bomar JD, Johnson KP, et al. Nonoperative management of femoroacetabular impingement: a prospective study. Am J Sports Med 2018;46:3415-22.

119 Schallmo MS, Fitzpatrick TH, Yancey HB, et al. Return-to-Play and performance outcomes of professional athletes in North America after hip arthroscopy from 1999 to 2016. Am J Sports Med 2018;46:1959-69.

120 Ishøi L, Thorborg K, Kraemer O, et al. The association between specific sports activities and sport performance following hip arthroscopy for femoroacetabular impingement syndrome: a secondary analysis of a cross-sectional cohort study including 184 athletes. J Hip Preserv Surg 2019;6:124-33.

121 Ishøi L, Thorborg K, Kemp JL, et al. Maximal hip muscle strength and rate of torque development 6-30 months after hip arthroscopy for femoroacetabular impingement syndrome: A cross-sectional study. J Sci Med Sport 2021. doi:10.1016/j. jsams.2021.05.006. [Epub ahead of print: 18 May 2021].

122 Casartelli NC, Bizzini M, Maffiuletti NA, et al. Exercise therapy for the management of femoroacetabular impingement syndrome: preliminary results of clinical responsiveness. Arthritis Care Res 2019;71:1074-83.

123 Guenther JR, Cochrane CK, Crossley KM, et al. A pre-operative exercise intervention can be safely delivered to people with femoroacetabular impingement and improve clinical and biomechanical outcomes. Physiother Can 2017;69:204-11.

124 Hunt $D$, Prather $H$, Harris Hayes M, et al. Clinical outcomes analysis of conservative and surgical treatment of patients with clinical indications of prearthritic, intraarticular hip disorders. Pm R 2012;4:479-87.

125 Emara K, Samir W, Motasem EH, et al. Conservative treatment for mild femoroacetabular impingement. J Orthop Surg 2011;19:41-5.

126 Coppack RJ, Bilzon JL, Wills AK, et al. Physical and functional outcomes following multidisciplinary residential rehabilitation for prearthritic hip pain among young active UK military personnel. BMJ Open Sport Exerc Med 2016;2:e000107.

127 Ometti M, Schipani D, Conte P, et al. The efficacy of intra-articular HYADD4-G injection in the treatment of femoroacetabular impingement: results at one year follow up. J Drug Assess 2020;9:159-66.

128 MECIR manual. Available: https://community.cochrane.org/mecir-manual [Accessed 8 Jul 2021].

129 Palmer AJR, Ayyar Gupta V, Fernquest S, et al. Arthroscopic hip surgery compared with physiotherapy and activity modification for the treatment of symptomatic femoroacetabular impingement: multicentre randomised controlled trial. BMJ 2019;364:1185. 